\title{
Modelling of flow boiling heat transfer in a cylindrical annulus mini gap
}

\author{
Magdalena Piasecka ${ }^{1,}$, Sylwia Hożejowska ${ }^{2}$ and Tomasz Musiał ${ }^{3}$ \\ ${ }^{1,3}$ Kielce University of Technology, Faculty of Mechatronics and Mechanical Engineering,Al. 1000-lecia P.P. 7, 25-314 Kielce, Poland \\ ${ }^{2}$ Kielce University of Technology, Faculty of Management and Computer Modelling, Al. 1000-lecia P.P. 7, 25-314 Kielce, Poland
}

\begin{abstract}
This paper presents the results of investigations into flow boiling heat transfer in a mini gap $1 \mathrm{~mm}$ wide. The mathematical approaches of the heat transfer coefficient determination were also proposed. It was considered two ways of describing layers in a measurement module with a cylindrical annulus mini gap. In the first approach, the measurement module was treated as a planar multilayer wall and in the second approach - as a multilayer cylindrical wall. The values of the local heat transfer coefficients, determined from two approaches, were compared. Both approaches gave similar results.
\end{abstract}

\section{Introduction}

In recent years, many theoretical and experimental works have been published to address the issues of heat transfer in flow boiling in mini gaps of different geometry and spatial orientation. The major advantage of mini gaps technology in heat exchangers is improving the effectiveness of heat transfer processes. Effective heat transfer is vital for the cooling processes in miniature equipment, as it prevents overheating and permanent damage to the components.

Studies devoted to the cooling technology that uses flow boiling processes in small channels will contribute to further development of current trends regarding the miniaturisation of products and devices. In [1,2], the authors present their investigations into heat transfer bubbly boiling process, harnessing environmentally friendly refrigerating media in conventional channels [1] and minichannels [2]. Research on heat transfer coefficients is reported in [3], where pressure drop and 'dryout' for flow boiling of water in an oil heated minichannel was discussed. In [4], the authors show an improved semi-empirical method for determining heat transfer coefficient in flow boiling in conventional and small diameter tubes. The issue of flow boiling heat transfer of R134a in the multiport minichannel heat exchangers is discussed in [5]. In [6], the study of R134a flow boiling heat transfer and evaluation of existing correlations was reported. In [7], in order to determine the heat transfer coefficient, the researches perform experimental tests during nanofluid (based on water and copper oxide particles) flow in a cylindrical channel. Paper [8] shows the results of experimental investigation into heat transfer of helium-xenon mixtures in cylindrical channels. In [9], the authors determine the heat transfer coefficient in a mini channel evaporator with R-134a as a refrigerant.
Experimental data were the basis for the analysis of flow boiling heat transfer in rectangular minichannels performed by Piasecka in her previous works [10-14]. This research interest is focused on flow boiling heat transfer in cylindrical annulus mini gap.

\section{Experimental stand}

The experimental setup shown in Fig.1, consists of several systems: the test loop in which the working fluid (distilled water) circulates, the supply and control system, the data and image acquisition system, and the lighting system.

The test loop is composed of: a gear pump, a compensating tank, a heat exchanger, a filter, mass flow meter, and a deaerator. The data and image acquisition system consists of: a high speed camera, a data acquisition station, a computer with special software, and the lighting.

Figure 2 presents the testing module with an annular mini gap. The mini gap $1 \mathrm{~mm}$ wide was created between the external tempered glass pipe for viewing flow patterns (1) and the copper pipe (2) positioned along the same axis. A cartridge heater (4) was located inside the pipe (2), axially and symmetrically, powered by autotransformer with adjusted current intensity. To fix the thermoelement sensors with a spacing of approximately $1 \mathrm{~cm}$ in the flow line longitudinal slots was made in the inside surface of the pipe. Thermoelement wires run in the gap between the copper pipe and the heater and in the thermal conductive filler layer (thermal paste). The module header had pressure transducers and the sensors of successive thermoelements to measure the water temperature and positive pressure in the inlet and the outlet to and from the mini gap.

${ }^{*}$ Corresponding author: tmpmi@tu.kielce.pl 
During experimental series, there was a laminar flow of distilled water along a mini gap. It was accompanied by a gradual increase in the electric power supplied to cartridge heater. The experiments were performed at the subcooled boiling region, in which the liquid flowing in the mini gap was superheated at the interface with the heater and subcooled at the core of the flow. The data from three experimental series performed at similar thermal and flow parameters, obtained for selected similar heat flux value, were used in the calculation and further analysis.

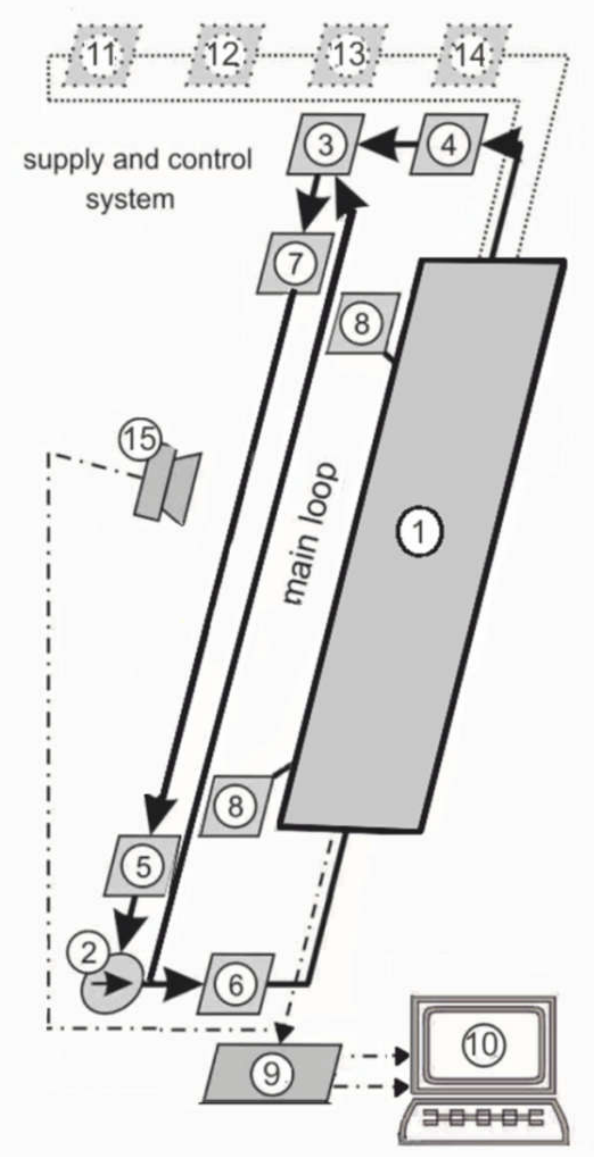

Fig. 1. The schematic diagram of the main loops at the experimental setup, 1-a measurement module with a mini gap; 2- a gear pump; 3- a compensating tank/pressure regulator; 4- a tube-type heat exchanger, 5- a filter, 6- a mass flow meter; 7- a deaerator; 8- a pressure transducer; 9- a data acquisition station; 10- a pc computer; 11- an inverter welder; 12- a voltmeter; 13- an ammeter; 14- a shunt;15- a high-speed camera.

\section{One-dimensional model}

It is assumed that the steady state heat transfer process in the test module is stationary. Since the width of the gap is relatively small in relation to the channel radius (Fig. 3), the elements of the module can be treated as a system of planar layers with different thickness and thermal conductivity. In paper notation, the subscripts referring to: the cartridge heater, the thermal conductive layer (thermal paste) with thermocouples, the copper pipe and the fluid in the mini gap are denoted with the following letters: $h$ for the cartridge heater, $s$ for the thermal conductive layer (thermal paste) with thermocouples, $F$ for the copper pipe and $f$ for the fluid in the mini gap. The cartridge heater (with radius $r_{l}$ ) is the heat source with power output $q_{V}$.

For $r=r_{1}$ at each point $z_{1}, z_{2}, \ldots, z_{M}$, the condition below is satisfied

$$
\frac{1}{2} r_{1} q_{V}=\lambda_{h} \frac{\partial T_{h}\left(r_{1}, z_{i}\right)}{\partial r}=\lambda_{s} \frac{\partial T_{s}\left(r_{1}, z_{i}\right)}{\partial r}
$$

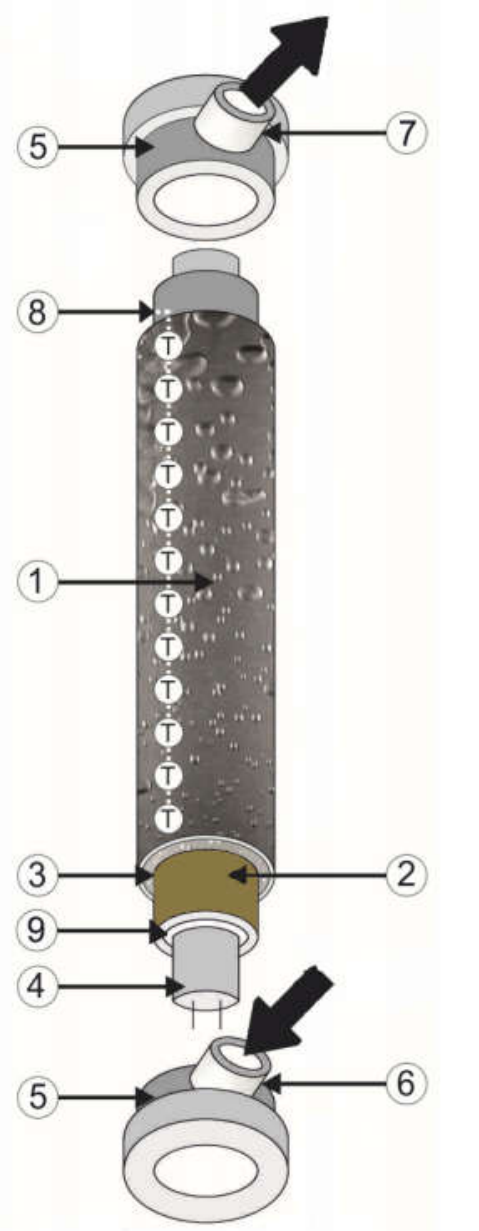

Fig. 2. The measurement module layout with an annular mini gap: 1- a glass pipe for viewing flow patterns, 2- a copper pipe, 3- an annular mini gap, 4- a cartridge heater, 5- a module header with sealing, 6- an inlet port for the medium, 7- an outlet port for the medium, 8-thermoelements between the heater (4) and the copper pipe (2) in the thermal conductive filler layer (thermal paste), 9- a thermal conductive layer (thermal paste).

Since difference $r_{2}-r_{1}$ is very small, it is possible to replace the partial derivative with a finite difference:

$$
\frac{\partial T_{s}\left(r_{1}, z_{i}\right)}{\partial r} \approx \frac{T_{s, i}-T_{h}\left(r_{1}, z_{i}\right)}{r_{2}-r_{1}}
$$

where $T_{s, i}=T_{s}\left(r_{2}, z_{i}\right)$ are the temperatures measured using the thermocouples at points $\left(r_{2}, z_{i}\right)$, Fig. $3 \mathrm{~b}$. 
a)

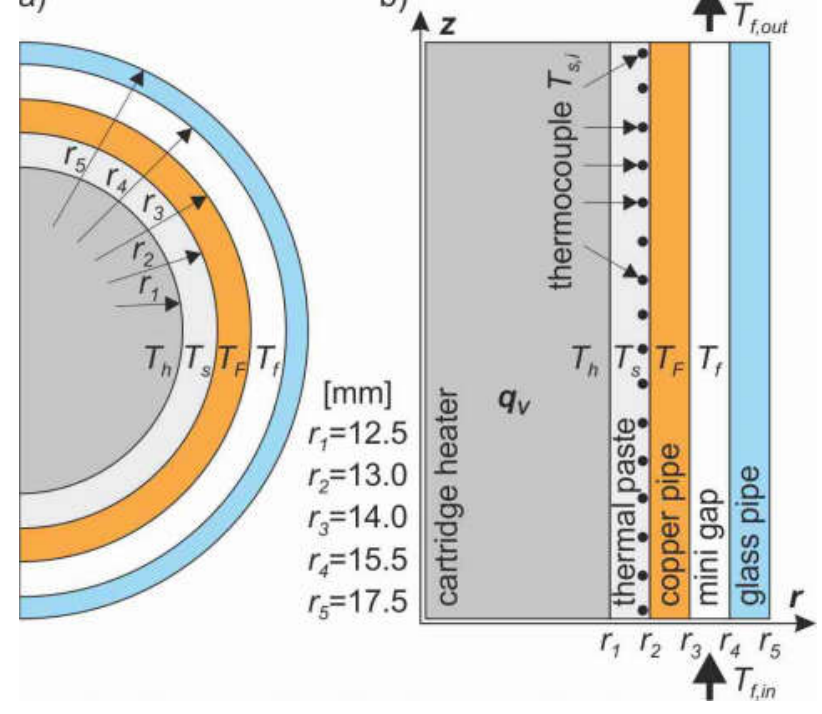

Fig. 3. Diagram illustrating the assumptions adopted for the planar multilayer wall: a) vertical cross-section, b) horizontal cross-section.

Combining equations (1) and (2) gives formula for the temperature $T_{h}\left(r_{1}, z_{i}\right)$ :

$$
T_{h}\left(r_{1}, z_{i}\right)=T_{s, i}+q_{V} \frac{\left(r_{2}-r_{1}\right) r_{1}}{2 \lambda_{s}}
$$

The relationship between the cartridge heater temperature and the copper pipe surface temperature for the planar multilayer wall (Fig. 3b) can be written as:

$$
T_{h}\left(r_{1}, z_{i}\right)-T_{F}\left(r_{3}, z_{i}\right)=\frac{q_{V} r_{1}}{2}\left(\frac{\left(r_{2}-r_{1}\right)}{\lambda_{s}}+\frac{\left(r_{3}-r_{2}\right)}{\lambda_{F}}\right)
$$

Equations (3) and (4) lead to formula

$$
T_{F}\left(r_{3}, z_{i}\right)=T_{s, i}-\frac{q_{V} r_{1}}{2} \frac{\left(r_{3}-r_{2}\right)}{\lambda_{F}}
$$

The formula for the local heat transfer coefficients at points $z_{1}, z_{2}, \ldots, z_{M}$, at the interface between the copper pipe surface and the fluid, derived from equation (5) and from the Robin boundary condition, has the form

$$
\alpha_{1}\left(z_{i}\right)=\alpha_{1, i}=\frac{0.5 r_{1} q_{V}}{T_{s, i}-0.5 r_{1} q_{V} \frac{\left(r_{3}-r_{2}\right)}{\lambda_{F}}-T_{f}}
$$

If it is assumed that the module elements create a cylindrical layer (Fig. 3a), formula (6) will have the following form

$$
\alpha_{2}\left(z_{i}\right)=\alpha_{2, i}=\frac{0.5 r_{1} q_{V}}{T_{s, i}-0.5 r_{1}^{2} q_{V} \frac{\ln \left(r_{3} / r_{2}\right)}{\lambda_{F}}-T_{f}}
$$

In formulas (6) and (7) it was assumed that the fluid temperature $T_{f}$ changes linearly in the mini gap, from the inlet temperature $T_{f, \text { in }}$ to the outlet temperature $T_{f, \text { out }}$. Both temperatures are known from the measurements.

\section{Results}

Results from the experiments performed at boiling heat transfer in the subcooled boiling region with distilled water as the working fluid flowing along a cylindrical annulus mini gap were presented. Selected results from three experimental series for the set similar heat flux values (approx. $62 \mathrm{MW} / \mathrm{m}^{2}$ on average) were used in two mathematical approaches for determining the heat transfer coefficient. In the first approach, the measurement module was treated as a planar multilayer wall and in the second approach - as a multilayer cylindrical wall. The results from the three different experiments performed at the set volumetric heat fluxes of $57.6\left[\mathrm{MW} / \mathrm{m}^{3}\right], 60.6\left[\mathrm{MW} / \mathrm{m}^{3}\right]$ and $66.7\left[\mathrm{MW} / \mathrm{m}^{3}\right]$ were analysed.

Figure 4 shows the heat transfer coefficient at the interface between the copper pipe and the water flowing in the mini gap, calculated from equation (6), i.e., using the calculation approach in which the measurement module was treated as a planar multilayer wall, vs. the mini gap length. It can be noticed that the heat transfer coefficient decreases, in the nearly monotonic mode, with the distance from the mini gap inlet. In all cases under analysis, local values of the heat transfer coefficient reached similar results, from the maximum value of $4\left[\mathrm{~kW} /\left(\mathrm{m}^{2} \mathrm{~K}\right)\right]$ at the inlet to the minimum value of $2.8\left[\mathrm{~kW} /\left(\mathrm{m}^{2} \mathrm{~K}\right)\right]$ at the outlet of the mini gap.

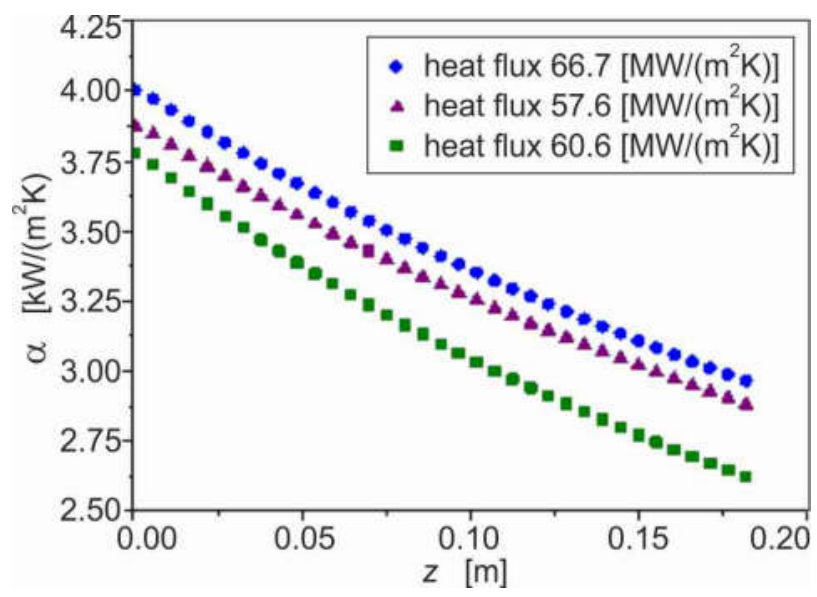

Fig. 4. Heat transfer coefficient vs. the mini gap length, calculated with the first approach, eq. 6 (the measurement module was treated as a planar multilayer wall).

The maximum relative error (MRE) describing the difference between the heat transfer coefficients obtained from equations (6) and (7), respectively, was calculated from the formula 


$$
\left.M R E=\max \left\{\frac{\sum_{i=1}^{M}\left(\alpha_{1}\left(z_{i}\right)-\alpha_{2}\left(z_{i}\right)\right)^{2}}{\sum_{i=1}^{M}\left(\alpha_{1}\left(z_{i}\right)\right)^{2}}\right]^{0.5},\left[\frac{\sum_{i=1}^{M}\left(\alpha_{1}\left(z_{i}\right)-\alpha_{2}\left(z_{i}\right)\right)^{2}}{\sum_{i=1}^{M}\left(\alpha_{2}\left(z_{i}\right)\right)^{2}}\right]^{0.5}\right\}
$$

In the three cases analysed (similar heat fluxes in the three experiments), the MRE was lower than $0.2 \%$. Local values of the heat transfer coefficient calculated from equation (7) (second calculation approach, in which the measurement module was treated as a multilayer cylindrical wall), take similar values, as that in Fig. 4, confirmed by very low MRE values.

\section{Conclusions}

The paper focuses on heat transfer modelling for the working fluid (distilled water) flowing in the symmetrically heated annular mini gap. In the mathematical approach presented here, two ways of describing the multilayer measurement module were used. In the first approach, measurement module was treated as a planar multilayer wall, and in the second approach - as a multilayer cylindrical wall. The calculation data for the heat transfer coefficient values were from three different experiments, with each of them performed with similar experimental thermal and flow parameters, including the most important one - heat flux.

The heat transfer coefficient calculated between the metal pipe and water in the mini gap took similar values in both approaches. The outcomes from the two methods allow concluding that both approaches give very similar results. This conclusion is confirmed analytically by a very low value of the maximum relative error (MRE), which in the cases under analysis was at most $0.2 \%$.

Further work will be focused on constructing a twodimensional heat transfer model in a mini annular gap and solving it with the use of CFD.

The research reported herein was supported in part by a grant from the National Science Centre in Poland

(No. DEC-2013/09/B/ST8/02825).

\section{References}

1. T. Bohdal, Ann. Set Environ. Prot. 15, 107-126. (2013)

2. T. Bohdal, Int. J. Heat Fluid Flow 21, 449-455 (2000)

3. L.A. Campbell, S.G. Kandlikar, Proc. Second Int. Conf. Microchannels Minichannels, June 17-19, Rochester (2004)

4. D. Mikielewicz, J. Mikielewicz, J. Tesmar, Int. J. Heat Mass Tran. 50, 3949-3956 (2007)

5. J. Kaew-On, K. Sakamatapan, S. Wongwises, Exp. Therm. Fluid Sci. 35, 364-374 (2011)

6. Y. Xu, X. Fang, G. Li, A. Xu, Int. J. Heat Mass Transf. 98, 150-163 (2016)

7. A.V. Minakov, V.Y. Rudyak, D.V. Guzei, A.S. Lobasov, High Temp. 53, 246-253 (2015)
8. S.L. Elistratov, O.V. Vitovskii, E.Y. Slesareva, J. Eng. Thermophys. 24, 33-35 (2015)

9. E.B. Dollera, E.P. Villanueva, IOP Conf. Ser. Mater. Sci. Eng. 88, 12027 (2015)

10. M. Piasecka, Exp. Heat Tran. 27, 231-255 (2014)

11. M. Piasecka, Heat Tran. Eng. 35 (10), 903-912 (2014)

12. M. Piasecka, Int. J. Heat Mass Tran. 81, 114-121 (2015)

13. M. Piasecka, Int. J. Refrig. 56, 198-212 (2015)

14. M. Piasecka, K. Strąk, B. Maciejewska, Heat Tran. Eng. 38, 332-346 (2017) 